\title{
Large cervicothoracic myxoinflammatory fibroblastic sarcoma with brachial plexus invasion: A case report and literature review
}

\author{
XIAOTIAN JIA* , JIANYUN YANG ${ }^{*}$, LIN CHEN and CONG YU \\ Department of Hand Surgery, Huashan Hospital, Fudan University, Shanghai 200040, P.R. China
}

Received February 3, 2015; Accepted June 16, 2016

DOI: $10.3892 / \mathrm{ol} .2016 .4824$

\begin{abstract}
Myxoinflammatory fibroblastic sarcoma is a rare sarcoma that develops in patients of all ages, which usually presents as a slow-growing painless mass in the distal extremities. To date, myxoinflammatory fibroblastic sarcoma with invasion of the brachial plexus has rarely been reported in the literature. In this study, a case of large cervicothoracic sarcoma, which invaded the brachial plexus, is presented. The patient reported no sensory disturbance or dyskinesia. The tumor was completely resected without injury of the brachial plexus. The postoperative histological diagnosis was myxoinflammatory fibroblastic sarcoma. Follow-up examination performed 24 months after surgery revealed no tumor recurrence and no sensory disturbance or dyskinesia was reported. This study presents a rare case of large myxoinflammatory fibroblastic sarcoma with brachial plexus invasion that was successfully managed by surgery.
\end{abstract}

\section{Introduction}

Myxoinflammatory fibroblastic sarcoma is a rare neoplasm, accounting for $1 \%$ of all adult malignancies (1). The tumor most commonly occurs in the distal extremities and usually affects males and females equally in the fourth and fifth decades of life. Myxoinflammatory fibroblastic sarcomas usually present as a slow-growing painless mass that can mimic infection, ganglion or benign tumors. Accurate diagnosis is based on the postoperative pathological result. Wide local excision is the first choice of treatment. For acral tumors with multiple recurrences, amputation may be considered. The efficacy of chemotherapy and radiotherapy

Correspondence to: Mr. Cong Yu, Department of Hand Surgery, Huashan Hospital, Fudan University, 12 Wu Lu Mu Qi Zhong Road, Shanghai 200040, P.R. China

E-mail: 875445584@qq.com

${ }^{*}$ Contributed equally

Key words: dyskinesia, myxoinflammatory fibroblastic sarcoma, cervicothoracic, brachial plexus, surgery, sensory disturbance remains unclear. Myxoinflammatory fibroblastic sarcoma is a low-grade sarcoma with a low rate of mortality and metastasis, demonstrating a long-term clinical course. However, the local recurrence rate is extremely high (2).

Tumors that invade the brachial plexus present a significant challenge as surgical procedures may lead to sensory disturbance and dyskinesia (2).

In this study, the rare case of large myxoinflammatory fibroblastic sarcoma with invasion of the brachial plexus, which that was successfully managed with surgery, is presented.

\section{Case report}

A 54-year-old man, who had suffered from a right cervicothoracic mass for nearly 16 years and did not obtain any medical treatment, was referred to the Department of Hand Surgery (Huashan Hospital, Fudan University, Shanghai, China) in January 2013. The patient presented with a huge mass with no pain and no sensory or motor dysfunction. The patient previously presented to Shanghai Changhai Hospital (Shanghai, China) in November 2012. Magnetic resonance imaging (MRI) and fine-needle aspiration was performed. Subsequently, the patient was referred from Shanghai Changhai Hospital to Huashan Hospital.

Physical examination revealed a right cervicothoracic mass that was palpable from the supraclavicular fossa to the infraclavicular region. The supraclavicular and infraclavicular portions of the mass were $\sim 10 \times 5 \times 3 \mathrm{~cm}$ and $25 \times 18 \times 8 \mathrm{~cm}$ in size, respectively. On palpation the mass was hard with no tenderness, clear boundaries and negativity for Tinel's sign. The active and passive activity of the right upper limb was normal and no abnormal sensation in the affected limb was reported.

B-ultrasound examination revealed a low echo-level mass located between the right neck and shoulder, extending from the right anterior chest wall to the right anterior axillary region. A chest radiograph identified a dense shadow at the right clavicular region. MRI revealed a large occupying lesion at the supraclavicular and infraclavicular region (Fig. 1). A pre-operative pathological examination performed at Shanghai Changhai Hospital in November 2012 indicated the diagnosis of a benign mesenchymal tumor.

The mass was extremely large, with a supraclavicular portion measuring $10 \times 5 \times 3 \mathrm{~cm}$ and an infraclavicular portion measuring $25 \times 18 \times 8 \mathrm{~cm}$ (Fig. 2). The mass was exposed via supraclavicular incision and the supraclavicular portion of the 


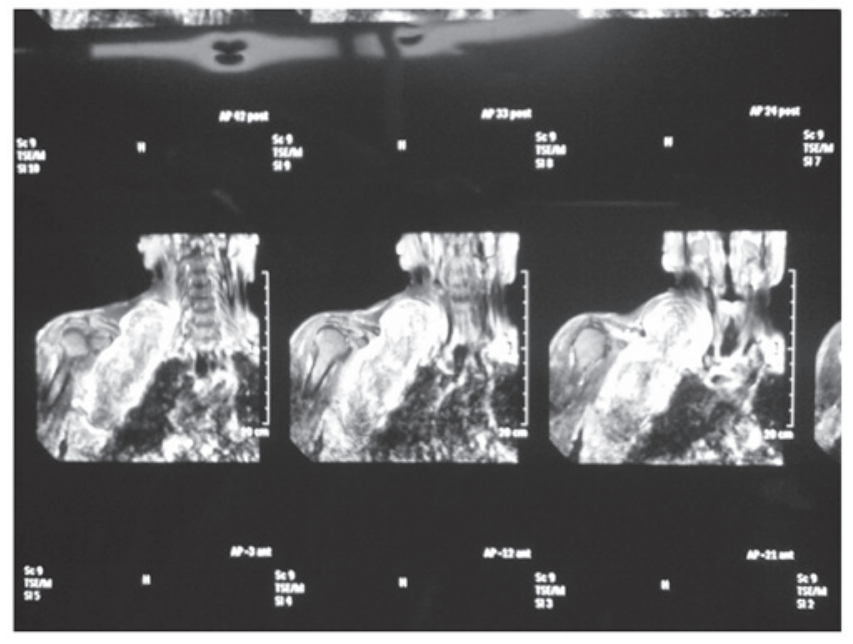

Figure 1. Magnetic resonance imaging revealing large occupying lesion at the supraclavicular and infraclavicular regions.



Figure 2. The supraclavicular portion of the mass was $10 \times 5 \times 3 \mathrm{~cm}$ and the infraclavicular portion of the mass was $25 \times 18 \times 8 \mathrm{~cm}$ prior to surgery.

hard mass, measuring $10 \times 5 \times 3 \mathrm{~cm}$, with clear boundaries and an abundant blood supply, was identified. The tumor oppressed the brachial plexus and vessels, and could not be separated by only supraclavicular incision. The mass extended from the supraclavicle to the infraclavicle and could not be completely exposed by only supraclavicular incision. An additional incision from the infraclavicle region to the deltopectoral interval and midaxillary line was made, and the infraclavicular portion of the hard mass, sized $25 \times 18 \times 8 \mathrm{~cm}$, with clear boundaries and an abundant blood supply, was identified. The tumor was lobulated. The mass was enwrapped and separated by the medial, lateral and posterior cords and the axillary, musculocutaneus, median and ulnar nerves (Fig. 3). The mass was evidently adhered to the aforementioned nerves and could not be easily separated. The mass was gradually resected carefully to maintain the integrity of the nerves (Fig. 4). Clavicotomy was performed prior to separation of the nerves and vessels surrounding the mass. Finally, the mass was completely resected (Fig. 5). Tumor invasion of the clavicle without bone destruction was observed. Intraoperative electromyography recorded the somatosensory evoked potential by stimulating

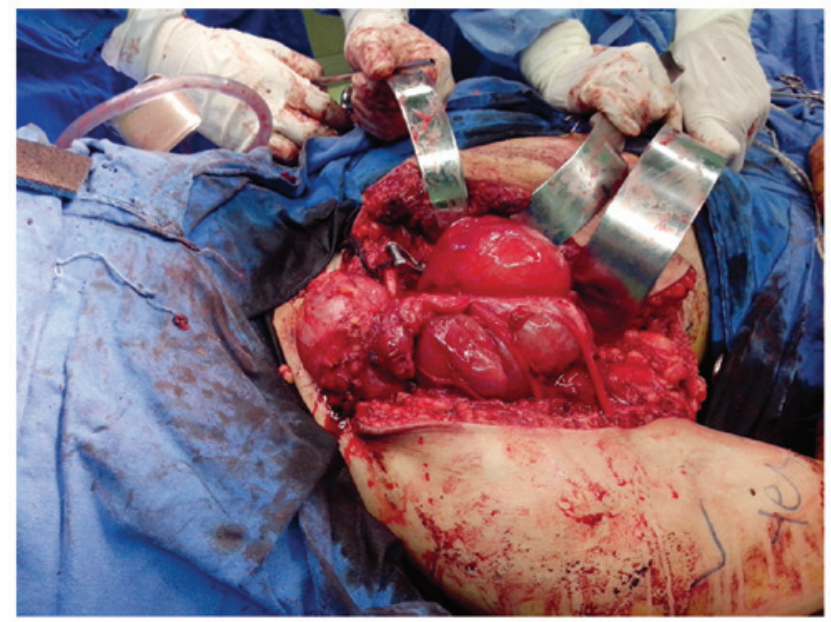

Figure 3. The mass was enwrapped and separated by the medial, lateral and posterior cords and the axillary, musculocutaneus, median and ulnar nerves.



Figure 4 . The mass was gradually resected to maintain the integrity of the nerves.

the axillary, musculocutaneus, median, radial and ulnar nerves. Following surgery, the activity and sensation of the right upper limb were normal.

The tissue used for histological analysis was embedded in paraffin and sectioned. The histological results showed a prominent mixed inflammatory infiltrate, macrophages, Touton-type giant cells and myxoid matrix, which confirmed the pathological diagnosis of myxoinflammatory fibroblastic sarcoma (Fig. 6) (3). Histopathologically, the lesion demonstrated numerous poly-morphonuclear leukocytes, each with a large vesicular nucleus. The tumor tissue was composed of atypical cells with boundaries that could not be clearly defined.

Immunostaining was performed by two certified pathologists in the present department who were blinded to the clinical characteristics of the patient. Micrometer-thick tissue sections were autoclaved in buffer, and incubated with antibodies. Immunostaining was performed using the biotin-free horseradish peroxidase enzyme-labeled polymer of the Envision Plus detection system. The antibodies used were as follows: anti-vimentin antibody (clone, EPR3776; catalog 




Figure 5. The mass was completely resected.



Figure 6. Postoperative histological results showing prominent mixed inflammatory infiltrate, macrophages, Touton-type giant cells and myxoid matrix, indicating a diagnosis of myxoinflammatory fibroblastic sarcoma.

no., ab92547; dilution, 1:200; $60 \mathrm{~min}$ at room temperature; Abcam, Cambridge, UK); anti-CD68 antibody (clone, KP1; catalog no., ab955; dilution, 1:200; $60 \mathrm{~min}$ at room temperature; Abcam); anti-CD34 antibody (clone, EP373Y; catalog no., ab81289; dilution, 1:250; $60 \mathrm{~min}$ at room temperature; Abcam); anti-S100 $\beta$ antibody (clone, EP1576Y; catalog no., ab52642; dilution, 1:500; $60 \mathrm{~min}$ at room temperature; Abcam); anti-cytokeratin antibody (clone, AE1/AE3; catalog no., M351501; dilution, 1:200; $60 \mathrm{~min}$ at room temperature; Dako, Glostrup, Denmark); anti-EMA antibody (clone, 2F6; catalog no., ab156947; dilution, 1:150; $60 \mathrm{~min}$ at room temperature; Abcam); and anti-Desmin antibody (clone, D33; catalog no., ab8470; dilution, 1:200; $60 \mathrm{~min}$ at room temperature; Abcam). The Periodic Acid Schiff (PAS) Stain kit (Baso, Zhuhai, China) was also used. Immunohistochemical staining revealed positivity for vimentin, periodic acid-Schiff, cluster of differentiation (CD)68, CD34, S100 and negativity for cytokeratin, epithelial membrane antigen and desmin.

Follow-up MRI examination 24-months after surgery revealed no evidence of tumor recurrence and no sensory disturbance or dyskinesia.
The patient was followed up every month in the first 3 months. Subsequently, the patient was followed up every 3 months until April 2016. Subsequently, the patient will be followed-up every 6 months.

\section{Discussion}

Myxoinflammatory fibroblastic sarcoma, originally termed 'acral myxoinflammatory fibroblastic sarcoma' was first identified by Meis-Kindblom and Kindblom (4) in 1998. In the same year, Montgomery et al (5) reported an inflammatory myxohyaline tumor of the distal extremities with virocyte or Reed-Sternberg-like cells, while Michal (6) reported inflammatory myxoid tumor of the soft parts with bizarre giant cells. In addition, Jurcić et al (7) demonstrated that occurrence of such tumors in the proximal regions of the limbs and thus the term 'myxoinflammatory fibroblastic sarcoma' was coined. Myxoinflammatory fibroblastic sarcoma develops in patients of all ages with no clear gender predilection.

Laskin et al (2) analyzed 104 myxoinflammatory fibroblastic sarcoma patients, which revealed that in $61 \%$ of cases, the tumor occurred in the fingers, hands and feet and in $73 \%$ of cases the tumor occurred in the dorsal soft tissue involving distal acral sites. Other affected regions included the knees and lower leg, elbow and forearm, ankle, upper leg, upper arm, shoulder and the inguinal region (2). To the best of our knowledge, the present case of a cervicothoracic myxoinflammatory fibroblastic sarcoma with brachial plexus invasion is the first to be reported in the literature. The largest myxoinflammatory fibroblastic sarcoma reported previously was $16 \mathrm{~cm}$ in size (8), while the supraclavicular and infraclavicular masses identified in this case were $10 \times 5 \times 3 \mathrm{~cm}$ and $25 \times 18 \times 8 \mathrm{~cm}$, respectively.

A painless slow-growing mass or swelling is the most common initial complaint in patients with myxoinflammatory fibroblastic sarcoma. A number of individuals also present with pain, tenderness or dysfunction of the affected area (8). The symptoms observed in the patient in the present case are consistent with those reported in the literature (2).

Preoperative auxiliary examinations indicated a benign tumor in the present study; however, postoperative histological analysis diagnosed low-grade malignant myxoinflammatory fibroblastic sarcoma. We hypothesize that in the context of diagnosis and treatment of large tumors, which are commonly benign, malignancy must always be suspected and therefore the use of pre-operative biopsy may improve diagnosis and treatment.

In the present case, the tumor was carefully resected to protect the medial, lateral and posterior cords, as well as the median, axillary and musculocutaneous nerves, which surrounded the tumor. The postoperative activity and sensation of the limb was normal. Surgery was difficult due to the large tumor size and invasion of the brachial plexus. Therefore, the tumor was separated carefully as injury of the brachial plexus may lead to dysfunction of the upper limb.

The histological differential diagnosis of myxoinflammatory fibroblastic sarcoma may be associated with the myxoid, inflammatory and atypical features. Differential diagnosis includes tenosynovitis, giant cell tumor of the tendon sheath, inflammatory myofibroblastic tumor, liposarcoma, epithelioid sarcoma and myxoid malignant fibrous histiocytoma (9). 
Lombardi et al (10) performed immunohistochemical analysis in 138 myxoinflammatory fibroblastic sarcoma patients, which revealed that vimentin was strongly positive in all lesions. A total of 84 and $57 \%$ of tumors exhibited focal positivity for CD68 and CD34, respectively. Focal positivity for smooth muscle actin, S-100 protein, activin receptor-like kinase 1 and keratin was also observed in $6-16 \%$ of patients (10). These results were consistent with the immunohistochemical staining results observed in the patient of the present case: Vimentin(+), CD68(+), CD34(+) and $\mathrm{S} 100(+)$.

Wide resection is generally accepted as the first choice of treatment for myxoinflammatory fibroblastic sarcoma. At present, the efficacy of chemotherapy and radiotherapy remains unclear and the rate of local recurrence is high $(11,12)$. In the present case, MRI performed during follow-up 24 months after surgery revealed no tumor recurrence. This case reported the successful surgical management of a huge myxoinflammatory fibroblastic sarcoma, which invaded the brachial plexus.

For such a large tumor with invasion of the brachial plexus, the neurological function of the brachial plexus may be preserved through a precise surgical procedure. The association between adjuvant therapy and the prognosis require observation in additional cases.

\section{References}

1. Silver AG, Baynosa RC, Mahabir RC, Wang WZ, Zamboni WA and Khiabani KT: Acral myxoinflammatory fibroblastic sarcoma: A case report and literature review. Can J Plast Surg 21: 92-94, 2013.
2. Laskin WB, Fetsch JF and Miettinen M: Myxoinflammatory fibroblastic sarcoma: A clinicopathologic analysis of 104 cases, with emphasis on predictors of outcome. Am J Surg Pathol 38: 1-12, 2014.

3. Nishio J: Updates on the cytogenetics and molecular cytogenetics of benign and intermediate soft tissue tumors. Oncol Lett 5: $12-18,2013$.

4. Meis-Kindblom JM and Kindblom LG: Acral myxoinflammatory fibroblastic sarcoma: A low-grade tumor of the hands and feet. Am J Surg Pathol 22: 911-924, 1998.

5. Montgomery EA, Devaney KO, Giordano TJ and Weiss SW: Inflammatory myxohyaline tumor of distal extremities with virocyte or Reed-Sternberg-like cells: A distinctive lesion with features simulating inflammatory conditions, Hodgkin's disease and various sarcomas. Mod Pathol 11: 384-391, 1998.

6. Michal M: Inflammatory myxoid tumor of the soft parts with bizarre giant cells. Pathol Res Pract 194: 529-533, 1998.

7. Jurcić V, Zidar A, Montiel MD, Frković-Grazio S, Nayler SJ, Cooper K, Suster S and Lamovec J: Myxoinflammatory fibroblastic sarcoma: A tumor not restricted to acral sites. Ann Diagn Pathol 6: 272-280, 2002.

8. Togral G, Arikan M, Aktas E and Gungor S: Giant myxoinflammatory fibroblastic sarcoma with bone invasion: A very rare clinical entity and literature review. Chin J Cancer 33: 406-410, 2014.

9. Lang JE, Dodd L, Martinez S and Brigman BE: Case reports: Acral myxoinflammatory fibroblastic sarcoma: A report of five cases and literature review. Clin Orthop Relat Res 445: 254-260, 2006.

10. Lombardi R, Jovine E, Zanini N, Salone MC, Gambarotti M, Righi A, Balladelli A, Colangeli M and Rocca M: A case of lung metastasis in myxoinflammatory fibroblastic sarcoma: Analytical review of one hundred and thirty eight cases. Int Orthop 37: 2429-2436, 2013.

11. Kovarik CL, Barrett T, Auerbach A and Cassarino DS: Acral myxoinflammatory fibroblastic sarcoma: Case series and immunohistochemical analysis. J Cutan Pathol 35: 192-196, 2008.

12. Tejwani A, Kobayashi W, Chen YL, Rosenberg AE, Yoon S, Raskin KA, Rosenthal DI, Nielsen GP, Hornicek FJ and Delaney TF: Management of acral myxoinflammatory fibroblastic sarcoma. Cancer 116: 5733-5739, 2010. 